\title{
Biomolecular structure refinement based on adaptive restraints using local-elevation simulation
}

\section{Journal Article}

\section{Author(s):}

Christen, Markus; Keller, Bettina; van Gunsteren, Wilfred F.

Publication date:

2007

Permanent link:

https://doi.org/10.3929/ethz-b-000003691

Rights / license:

In Copyright - Non-Commercial Use Permitted

Originally published in:

Journal of Biomolecular NMR 39(4), https://doi.org/10.1007/s10858-007-9194-2 


\title{
Biomolecular structure refinement based on adaptive restraints using local-elevation simulation
}

\author{
Markus Christen · Bettina Keller · Wilfred F. van Gunsteren
}

Received: 2 April 2007/ Accepted: 30 July 2007/Published online: 11 October 2007

(C) Springer Science+Business Media B.V. 2007

\begin{abstract}
Introducing experimental values as restraints into molecular dynamics (MD) simulation to bias the values of particular molecular properties, such as nuclear Overhauser effect intensities or distances, dipolar couplings, ${ }^{3} J$-coupling constants, chemical shifts or crystallographic structure factors, towards experimental values is a widely used structure refinement method. Because multiple torsion angle values $\phi$ correspond to the same ${ }^{3} J$-coupling constant and high-energy barriers are separating those, restraining ${ }^{3} J$-coupling constants remains difficult. A method to adaptively enforce restraints using a local elevation (LE) potential energy function is presented and applied to ${ }^{3} J$-coupling constant restraining in an MD simulation of hen egg-white lysozyme (HEWL). The method succesfully enhances sampling of the restrained torsion angles until the 37 experimental ${ }^{3} J$-coupling constant values are reached, thereby also improving the agreement with the 1,630 experimental NOE atom-atom distance upper bounds. Afterwards the torsional angles $\phi$ are kept restrained by the built-up local-elevation potential energies.
\end{abstract}

Keywords Molecular dynamics simulation . ${ }^{3} J$-coupling constant $\cdot$ Local-elevation search . Restrained simulation - Hen egg white lysozyme . Gromos $\cdot$ Force field $\cdot$ Structure determination

M. Christen · B. Keller · W. F. van Gunsteren ( $₫)$ Laboratory of Physical Chemistry, Swiss Federal Institute of Technology Zürich, ETH-Zürich, 8093 Zürich, Switzerland e-mail:wfvgn@igc.phys.chem.ethz.ch

\section{Introduction}

Experimental techniques such as X-ray diffraction and NMR spectroscopy are widely used to derive structural information from molecules in solution, solid state or in crystal form. These experimental methods have in common that the values of observable quantities are averages over time and over an ensemble of molecules. It may even not be possible to come up with a single physically plausible structure or conformation reproducing all experimental values (Jardetzky 1980; Nanzer et al. 1994). Therefore, the corresponding properties of an MD simulation should be calculated as time averages and when restraints are applied, those should reproduce the experimental values on average (Torda et al. 1989). The latter can be achieved by adding a penalty function $V^{\text {restr }}$ to the physical force field $V^{\text {phys }}$ of the MD simulation (Kaptein et al. 1985),

$V(\mathbf{r}(t))=V^{\text {phys }}(\mathbf{r}(t))+V^{\text {restr }}(\mathbf{r}(t))$,

using a penalty function $V^{\text {restr }}$ of the form (Scott et al. 1998)

$V^{\mathrm{restr}}(\mathbf{r}(t))=\sum_{k=1}^{N_{\text {restr }}} 1 / 2 K_{k}^{q r}\left(q_{k}(\mathbf{r}(t))-q_{k}^{0}\right)^{2}\left(\overline{q_{k}(\mathbf{r}(t))}-q_{k}^{0}\right)^{2}$,

where $\overline{q(\mathbf{r}(t))}$ may be a weighted average during the simulation (Torda et al. 1989) and $q(\mathbf{r}(t))$ is any of the above mentioned observables (van Gunsteren et al. 1999). By introduction of the first quadratic factor of Eq. 2, the functional form given here avoids generating large artificial structural fluctuations, as observed when using standard time-averaging ${ }^{3} J$-value restraints (Torda et al. 1993; Nanzer et al. 1997). 
The ${ }^{3} J$-coupling constants are usually calculated using the Karplus relation (Karplus 1959)

$J(\theta(\mathbf{r}(t)))=a \cos ^{2} \theta+b \cos \theta+c$,

where $\theta$ is the torsion angle defined by the four covalently bound atoms that determine a particular ${ }^{3} J$-coupling constant. This relation is of approximative nature and the constants $a, b$ and $c$ are generally calibrated by fitting measured ${ }^{3} \mathrm{~J}$-values for molecules whose dihedral angles are known from crystal structures (de Marco et al. 1978; Pardi et al. 1984; Brüschweiler and Case 1994; Wang and Bax 1996) or infered from NMR data (Schmidt et al. 1999). Since this Karplus relation is multi-valued for almost all except the very large and very small ${ }^{3} J$ values and the average $\overline{{ }^{3} J(\theta(\mathbf{r}))}$ is very nonlinear with respect to the average in $\theta$, restraining using a standard penalty function may lead to unrealistic results (Scott et al. 1998; van Gunsteren et al. 1999; Nanzer et al. 1997). Moreover, highenergy barriers between different conformations or $\theta$-angle values may inhibit a proper sampling of the various $\theta$-angle ranges that contribute to the measured averaged ${ }^{3} J$-values. These features of the relation between ${ }^{3} J$-values and dihedral angles have made their use in biomolecular structure refinement problematic. Here a solution to this problem is proposed.

In the next section, the new restraining method is explained, followed by an application of ${ }^{3} J$-value restraining to hen egg-white lysozyme and by a short discussion.

\section{Theory}

During a molecular dynamics simulation, the current (instantaneous) and average ${ }^{3} J$-coupling constants can be monitored. For this, the ${ }^{3} J$-values are expressed in terms of dihedral angles $\phi$ that are defined by non-hydrogen atoms of the molecule. Such an angle $\phi$ differs by a phase shift $\delta$ from the angle $\theta(\theta=\phi+\delta$, for the precise definition used by us see (van Gunsteren et al. 1996; Scott et al. 1999)). The average is calculated using an exponentially decaying memory function, which results in a larger impact of recent ${ }^{3} J$-values on the average,

$\bar{J}(t, \tau)=\frac{1}{\tau} \frac{1}{1-\exp (-t / \tau)} \int_{0}^{t} \exp \left(-\frac{t^{\prime}-t}{\tau}\right) J\left(t^{\prime}\right) \mathrm{d} t^{\prime}$

with $\tau$ the memory relaxation time (which determines how fast the memory decays) and ${ }^{3} J(t)$ the calculated ${ }^{3} J$-value at time $t$ (Torda et al. 1989, 1993). If the average (Eq. 4) and the experimental ${ }^{3} \mathrm{~J}$-value do not match, a local (limited range) potential energy term for the dihedral angle corresponding to the particular ${ }^{3} J$-coupling constant is introduced and increased in size until the dihedral angle changes value. In other words, as long as the calculated and experimental ${ }^{3} J$-values do not match, the dihedral angle is forced away from the range of values that were sampled up till now in the simulation. This idea derives from localelevation (le) search (Huber et al. 1994) in which the potential energy of already visited parts of configuration space is raised in order to avoid repetitive sampling of the same parts of configuration space in the simulation.

The mathematical and algorithmic formulation of the proposed method is the following. Whenever the simulated average of the ${ }^{3} J$-value and the current ${ }^{3} J$-value do not fulfill the experimental observation, the force constant of a penalty function, acting on the torsion angle $\phi$ and its current value $\phi(t)$, is increased. The restraining potential energy function of a given $(k \mathrm{th}){ }^{3} J$-value is a sum of $N_{\mathrm{le}}$ (local) terms

$V_{k}^{\mathrm{Jres}}\left(\phi_{k}(\mathbf{r}(t))\right)=\sum_{i=1}^{N_{\mathrm{le}}} V_{k i}^{\mathrm{le}}\left(\phi_{k}(t)\right)$,

where, as in local-elevation conformational search (Huber et al. 1994), Gaussian functions centred at $\phi_{k i}^{0}$ are used as (locally active, i.e. only around $\phi^{0}$ ) penalty terms:

$V_{k i}^{\mathrm{le}}\left(\phi_{k}(\mathbf{r}(t))\right)=K^{\mathrm{Jres}} w_{\phi_{k i}}(t) \exp \left(-\left(\phi_{k}(t)-\phi_{k i}^{0}\right)^{2} / 2\left(\Delta \phi^{0}\right)^{2}\right)$,

where $w_{\phi_{k i}}(t)$ is the weight of the $i$ th penalty function and $K^{\mathrm{Jres}}$ the penalty function force constant. The centres $\phi_{k i}^{0}$ of the Gaussian functions $V_{k i}^{\mathrm{le}}$ are equally distributed over the range of possible values of $\phi_{k}\left(\phi_{k i}^{0}=2 \pi i / N_{\text {le }}\right.$ with $i=1, \ldots$, $N_{\text {le }}$ ), and the width is given by $\Delta \phi^{0}=2 \pi / N_{\text {le }}$.

The weight of the penalty function is accumulated during the simulation according to

$$
\begin{aligned}
w_{\phi_{k i}}(t)= & t^{-1} \int_{0}^{t} \delta_{\phi_{k}\left(\mathbf{r}\left(t^{\prime}\right)\right) \phi_{k i}^{0}}\left(J\left(\phi_{k}\left(\mathbf{r}\left(t^{\prime}\right)\right)\right)-J_{k}^{0}\right)^{2} \\
& \left(\bar{J}\left(\phi_{k}\left(\mathbf{r}\left(t^{\prime}\right)\right)\right)-J_{k}^{0}\right)^{2} \mathrm{~d} t^{\prime}
\end{aligned}
$$

using a biquadratic term (Scott et al. 1998) $\left(J\left(\phi_{k}(t)\right)-J_{k}^{0}\right)^{2}$ $\left(\bar{J}\left(\phi_{k}(t)\right)-J_{k}^{0}\right)^{2}$ to determine whether the ${ }^{3} J$-value deviates from the experimentally observed one $\left(J_{k}^{0}\right)$ with $\phi_{k}(\mathbf{r}(t))$ being the torsion angle corresponding to the ${ }^{3} J$-coupling constant $J_{k}$ and $\delta$ the Kronecker delta, which is defined using finite differences:

$\delta_{\phi_{k}(t) \phi_{k i}^{0}}= \begin{cases}1 & \text { if } \phi_{k i}^{0}-\Delta \phi^{0} / 2 \leq \phi_{k}(t)<\phi_{k i}^{0}+\Delta \phi^{0} / 2 \\ 0 & \text { otherwise. }\end{cases}$

Equation 7 ensures that the conformation is pushed away from $\phi_{k i}^{0}$ unless either the average $\bar{J}\left(\phi_{k}(t)\right)$ or the 
current value $J\left(\phi_{k}(t)\right)$ are close to the experimental one, which leads sooner or later to an average close to the experimental ${ }^{3} J_{k}$-value $J_{k}^{0}$.

It is straightforward to calculate the force resulting from $V_{k}^{\mathrm{Jres}}$ on particle $q$ :

$$
\begin{aligned}
\mathbf{f}_{q} & =-\frac{\partial}{\partial \mathbf{r}_{q}} V_{k}^{\mathrm{Jres}}=-\sum_{i=1}^{N_{\mathrm{le}}} \frac{\partial}{\partial \phi_{k}} V_{k i}^{\mathrm{le}} \frac{\partial \phi_{k}}{\partial \mathbf{r}_{q}} \\
& =\sum_{i=1}^{N_{\mathrm{le}}} V_{k i}^{\mathrm{le}} \frac{\left(\phi_{k}-\phi_{k i}^{0}\right)}{\left(\Delta \phi^{0}\right)^{2}} \frac{\partial \phi_{k}}{\partial \mathbf{r}_{q}} .
\end{aligned}
$$

Contrary to the original local-elevation method (Huber et al. 1994), using penalty functions to enforce restraints does not suffer from combinatorial explosion with increasing number of local-elevation degrees of freedom, as all restraints are treated independently.

In practice, flat bottom restraining can be achieved by only increasing the penalty function force constants $w_{\phi k i}(t)$ if the ${ }^{3} J$-value deviates more than a given value $\Delta J^{0}$ from the experimental value $J_{k}^{0}$. For the instantaneous factor of the penalty function this leads to

$$
\begin{aligned}
& V^{\text {inst,Jrest }}\left(J\left(\phi_{k}(t)\right)\right) \\
& = \begin{cases}\left(J\left(\phi_{k}(t)\right)-J_{k}^{0}-\Delta J^{0}\right)^{2} & \text { for } J\left(\phi_{k}(t)\right)>J_{k}^{0}+\Delta J^{0} \\
\left(J\left(\phi_{k}(t)\right)-J_{k}^{0}+\Delta J^{0}\right)^{2} & \text { for } J\left(\phi_{k}(t)\right)<J_{k}^{0}-\Delta J^{0} \\
0 & \text { otherwise, }\end{cases}
\end{aligned}
$$

and accordingly for the time-averaging factor

$$
\begin{aligned}
& V^{\text {avg,Jrest }}\left(\bar{J}\left(\phi_{k}(t)\right)\right) \\
& = \begin{cases}\left(\bar{J}\left(\phi_{k}(t)\right)-J_{k}^{0}-\Delta J^{0}\right)^{2} & \text { for } \bar{J}\left(\phi_{k}(t)\right)>J^{0}+\Delta J^{0} \\
\left(\bar{J}\left(\phi_{k}(t)\right)-J_{k}^{0}+\Delta J^{0}\right)^{2} & \text { for } \bar{J}\left(\phi_{k}(t)\right)<J^{0}-\Delta J^{0} \\
0 & \text { otherwise. }\end{cases}
\end{aligned}
$$

\section{Methods and results}

The protein hen egg-white lysozyme was recently used to validate (Oostenbrink et al. 2005; Soares et al. 2004) the Gromos (Christen et al. 2005; Scott et al. 1999; van Gunsteren et al. 1996) 53A6 force field (Oostenbrink et al. 2004), and the 45A3 parameter set (Schuler et al. 2001). Among the experimental data used was a set of hundred ${ }^{3} J_{\alpha \beta}$-coupling constants (Smith et al. 1991). Here only a subset of $37{ }^{3} J_{\alpha \beta}$-coupling constants that were assigned stereospecifically (Smith et al. 1991), which can therefore be used in ${ }^{3} J$-value restraining, were considered (see Table 1 ). We note that it is the $\chi_{1}$ side-chain torsional angle that plays the role of the restrained angle $\phi$ in Eqs. 5-11. Short simulations in vacuo using the X-ray structure as starting configuration showed $14^{3} \mathrm{~J}$-coupling constants with a deviation higher than $2 \mathrm{~Hz}$. Trying to reduce the deviation from experiment using ${ }^{3} J$-value restraining was only partially successful. We did not succeed in finding a good value of the force constant for any one of the restraining methods (instantaneous, time-averaged or biquadratic restraining penalty function) that would bring all ${ }^{3} J$-coupling constants close to the experimental values without seriously changing the secondary structure. Two issues needed to be addressed: First, to be able to use a minimal restraining force constant, the latter should be adjusted individually for each ${ }^{3} J$-value restraint. Second, to reproduce the experimental ${ }^{3} J$-value and escape local minima of the physical or restraint potential energy surface enhanced sampling of the corresponding torsional angle may be required. Adaptive restraints using local-elevation satisfy both requirements, as the force constant of the restraining penalty function is, if necessary, slowly built up during the simulation for each restraint. Furthermore, the restraining is achieved by pushing the simulation away from already visited conformations with ${ }^{3} J$-coupling constants different from the experimental ones. In other words, sampling is enhanced for dihedral angle degrees of freedom with wrong ${ }^{3} J$-values.

First, the sensitivity of the method with regard to the parameters $K^{\mathrm{Jres}}, \Delta J^{0}$ and $N_{\text {le }}$ was investigated. From a short 100 ps unrestrained simulation of lysozyme in vacuo, using a time step size of 2 fs and constraining bond lengths by the SHAKE (Ryckaert et al. 1977) algorithm, an average violation of 1,630 NOE distance upper bounds (Soares et al. 2004; Schwalbe et al. 2001) of $0.016 \mathrm{~nm}$ and a rootmean-square deviation (RMSD) for the 37 selected ${ }^{3} J_{\alpha \beta}$-coupling constants of $3.2 \mathrm{~Hz}$ were obtained. Then, a total of 36 simulations, each starting from the X-ray structure and lasting $100 \mathrm{ps}$, with all combinations of values for $K^{\mathrm{Jres}}=0.1,0.05,0.01,0.005 \mathrm{~Hz}^{-4}, \Delta J^{0}=0.5$, $0.75,1.0 \mathrm{~Hz}$ and $\Delta \phi^{0}=30^{\circ}, 20^{\circ}, 10^{\circ}\left(N_{\mathrm{le}}=12,18,36\right)$, were used to determine whether significant improvement in the RMSD for the ${ }^{3} J_{\alpha \beta}$-coupling constants could be obtained without disrupting the structure, measured by the average violation of 1,630 experimental NOE distance upper bounds (see Fig. 1). Colours in the figure correspond to equal force constants (red for $K^{\mathrm{Jres}}=0.1 \mathrm{~Hz}^{-4}$, blue for $0.05 \mathrm{~Hz}^{-4}$, yellow for $0.01 \mathrm{~Hz}^{-4}$ and green for $0.005 \mathrm{~Hz}^{-4}$ ), and line-styles to an equal number of intervals (solid for 12 intervals, dashed for 18 and dotted for 36) or local-elevation Gaussians per dihedral angle. The three values connected by a line use, from low to high ${ }^{3} J$-value RMSD, an allowed deviation of $\Delta J^{0}=0.5 \mathrm{~Hz}$ for the first, $\Delta J^{0}=$ $0.75 \mathrm{~Hz}$ for the second and $\Delta J^{0}=1.0 \mathrm{~Hz}$ for the last value. Using 36 intervals, all simulations did better or equal in NOE violations (the value of the unrestrained simulation is 
Table 1 Subset of $37{ }^{3} J_{\alpha \beta}$-coupling constants (in Hz) which could be assigned stereospecifically for hen-egg white lysozyme (Smith et al. 1991) and are used in (local-elevation) ${ }^{3} J$-value restraining

\begin{tabular}{|c|c|c|c|c|c|c|c|}
\hline \multicolumn{3}{|c|}{ Residue } & \multirow[t]{2}{*}{${ }^{3} J^{0}$} & \multicolumn{3}{|c|}{ Residue } & \multirow[t]{2}{*}{${ }^{3} J^{0}$} \\
\hline Name & Number & Proton & & Name & Number & Proton & \\
\hline Val & 2 & $\beta$ & 10.8 & Phe & 3 & $\beta_{3}$ & 3.0 \\
\hline Cys & 6 & $\beta_{2}$ & 11.5 & His & 15 & $\beta_{2}$ & 11.2 \\
\hline Asp & 18 & $\beta_{3}$ & 11.0 & Tyr & 20 & $\beta_{3}$ & 11.7 \\
\hline Tyr & 23 & $\beta_{2}$ & 10.9 & Asn & 27 & $\beta_{2}$ & 10.3 \\
\hline Val & 29 & $\beta$ & 11.1 & Cys & 30 & $\beta_{2}$ & 5.3 \\
\hline Phe & 34 & $\beta_{3}$ & 5.0 & Asn & 39 & $\beta_{2}$ & 4.5 \\
\hline Thr & 40 & $\beta$ & 4.5 & Thr & 43 & $\beta$ & 3.7 \\
\hline Asn & 46 & $\beta_{3}$ & 4.7 & Thr & 47 & $\beta$ & 2.6 \\
\hline Asp & 48 & $\beta_{2}$ & 2.6 & Thr & 51 & $\beta$ & 9.3 \\
\hline Asp & 52 & $\beta_{2}$ & 11.6 & Tyr & 53 & $\beta_{2}$ & 10.4 \\
\hline Asn & 59 & $\beta_{2}$ & 5.4 & Arg & 61 & $\beta_{3}$ & 10.8 \\
\hline Asp & 66 & $\beta_{3}$ & 4.5 & Thr & 69 & $\beta$ & 9.3 \\
\hline Leu & 75 & $\beta_{3}$ & 2.1 & Asp & 87 & $\beta_{2}$ & 5.1 \\
\hline Ile & 88 & $\beta$ & 4.5 & Thr & 89 & $\beta$ & 9.5 \\
\hline Val & 92 & $\beta$ & 10.1 & Cys & 94 & $\beta_{2}$ & 4.0 \\
\hline Val & 99 & $\beta$ & 6.3 & Val & 109 & $\beta$ & 8.0 \\
\hline Thr & 118 & $\beta$ & 4.2 & Asp & 119 & $\beta_{2}$ & 4.9 \\
\hline Trp & 123 & $\beta_{2}$ & 10.6 & Ile & 124 & $\beta$ & 4.6 \\
\hline Cys & 127 & $\beta_{2}$ & 11.6 & & & & \\
\hline
\end{tabular}

The $\beta_{2}$ and $\beta_{3}$ protons are defined according to standard rules (Markley et al. 1998). The experimental error is about $1 \mathrm{~Hz}$ (Smith et al. 1991)

indicated by the dotted black line), and even with an allowed deviation of $\Delta J^{0}$ of $1 \mathrm{~Hz}$, satisfactory ${ }^{3} J_{\alpha \beta^{-c o u}}$ pling constants were obtained. Using sufficiently small force constants and enough intervals resulted in lower average NOE upper bound violations.

In Fig. 2 the root-mean-square deviation over the set of 37 selected ${ }^{3} J$-coupling constants during $100 \mathrm{~ns}$ of MD simulation of lysozyme in vacuo is shown. The solid black line, denoting an unrestrained simulation, shows an increase in deviation from experiment. All other lines correspond to adaptively restrained simulations, the thin line with a force constant $K^{\mathrm{Jres}}$ of $0.005 \mathrm{~Hz}^{-4}$ and an acceptable deviation of $\Delta J^{0}=1.0 \mathrm{~Hz}$, the dashed one with a force constant of $0.1 \mathrm{~Hz}^{-4}$ and an acceptable deviation of $0.5 \mathrm{~Hz}$ and the dotted on with $K^{\mathrm{Jres}}=0.05 \mathrm{~Hz}^{-4}$ and $\Delta J^{0}=$ $0.75 \mathrm{~Hz}$. All use 36 intervals to discretize $\phi\left(N_{\mathrm{le}}=36\right)$. The time-averaging memory relaxation time $\tau$ (Eq. 4) used in all restrained simulations was 5 ps. (Torda et al. 1993; Nanzer et al. 1995). All combinations of parameters improve the RMSD of the ${ }^{3} J$-coupling constants within the first $30 \mathrm{ps}$. The longer the simulation is, the lower the force constant $K^{\mathrm{Jres}}$ may be to perturb the system as little as possible.

Comparing the evolution of selected angles $\phi$ during the simulation, three observations can be made: First, when starting from a configuration with a ${ }^{3} J$-coupling constant far from the experimental value, rotation around the corresponding dihedral angle is immediate. An example is shown in Fig. 3, panel C. The upper half shows the restraining potential energy after 100 ps simulation time, the lower half the time series of the corresponding dihedral angle (black dots denote the adaptively restrained, red dots the unrestrained simulation). It represents ${ }^{3} J_{\alpha \beta}$ of $\operatorname{Thr}(89)$, with an experimental value of $J^{0}=9.5 \mathrm{~Hz}$. The unrestrained simulation results in an average of $2.5 \mathrm{~Hz}$ whereas the adaptively restrained simulation gives $9.9 \mathrm{~Hz}$ (see also Table 2). Second, enhanced sampling until the ${ }^{3} J$ value matches the experimental data, or permanently, if the experimental value is an average over two (or more) states, is achieved (panel $\mathrm{D}:{ }^{3} J_{\alpha \beta}$ of $\operatorname{Val}(109)$, experimental: 8.0 $\mathrm{Hz}$, unrestrained: $3.2 \mathrm{~Hz}$, restrained $8.2 \mathrm{~Hz}$; panel B: ${ }^{3} J_{\alpha \beta}$ of $\operatorname{Thr}(69)$, experimental: $9.3 \mathrm{~Hz}$, unrestrained: 12.5 $\mathrm{Hz}$, restrained $9.8 \mathrm{~Hz}$ ). And third, if the ${ }^{3} J$-value is close to the experimental one from the beginning, the corresponding dihedral angle is kept restrained to its value (panel A: ${ }^{3} J_{\alpha \beta}$ of $\operatorname{Thr}(51)$, experimental: $9.3 \mathrm{~Hz}$, unrestrained $4.3 \mathrm{~Hz}$, restrained: $9.7 \mathrm{~Hz}$ ). The root-mean-square fluctuation of the torsion angle $\phi$, once the correct conformation is found, is in the same order of magnitude for the restrained as for the unrestrained simulations.

Using restraints, the atom-positional root-mean-square deviation from the initial (X-ray) structure (considering 


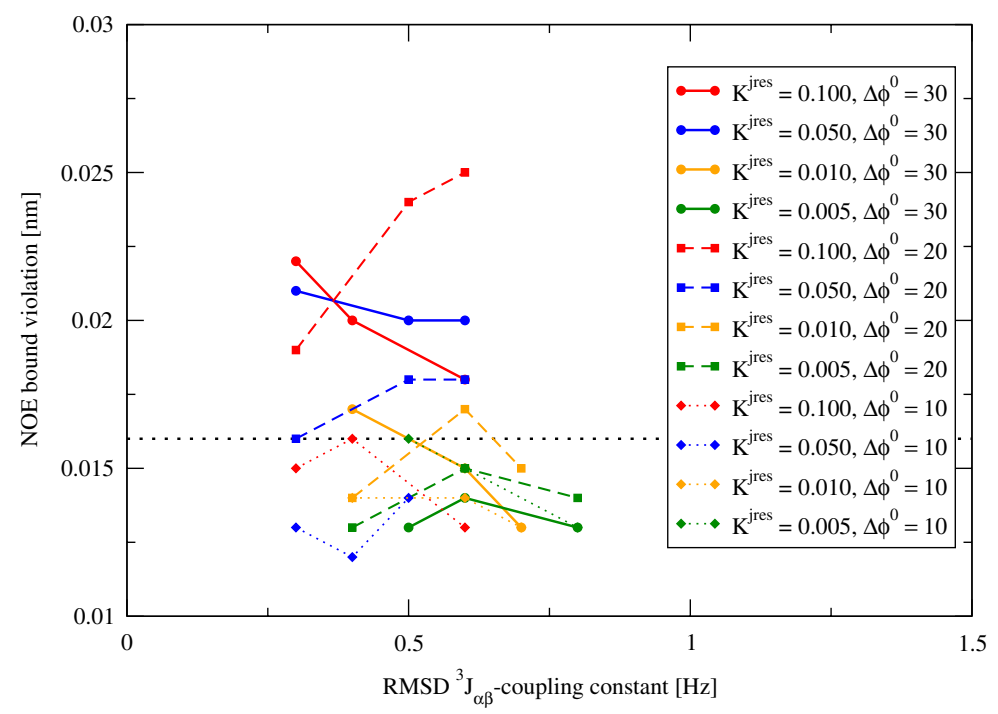

Fig. 1 Average of the NOE distance upper bound violations as a function of the root-mean-square deviation (RMSD) of a set of 37 selected (see Table 1) ${ }^{3} J_{\alpha \beta}$-coupling constants from experimental values for different force constants $K^{\mathrm{Jres}}=0.100,0.050,0.010,0.005 \mathrm{~Hz}^{-4}$ and different number of intervals $N_{\mathrm{le}}=12,18,36$ with corresponding $\Delta \phi^{0}=$
30, 20, 10 degree. On each line the first value represents an allowed deviation of $\Delta J^{0}=0.50 \mathrm{~Hz}$, the second $\Delta J^{0}=0.75 \mathrm{~Hz}$ and the third $\Delta J^{0}=$ $1.00 \mathrm{~Hz}$. The average NOE bound violation of the free simulation is indicated by the dotted black line. The RMSD of the ${ }^{3} J_{\alpha \beta}$-coupling constant in the free simulation is $3.2 \mathrm{~Hz}$

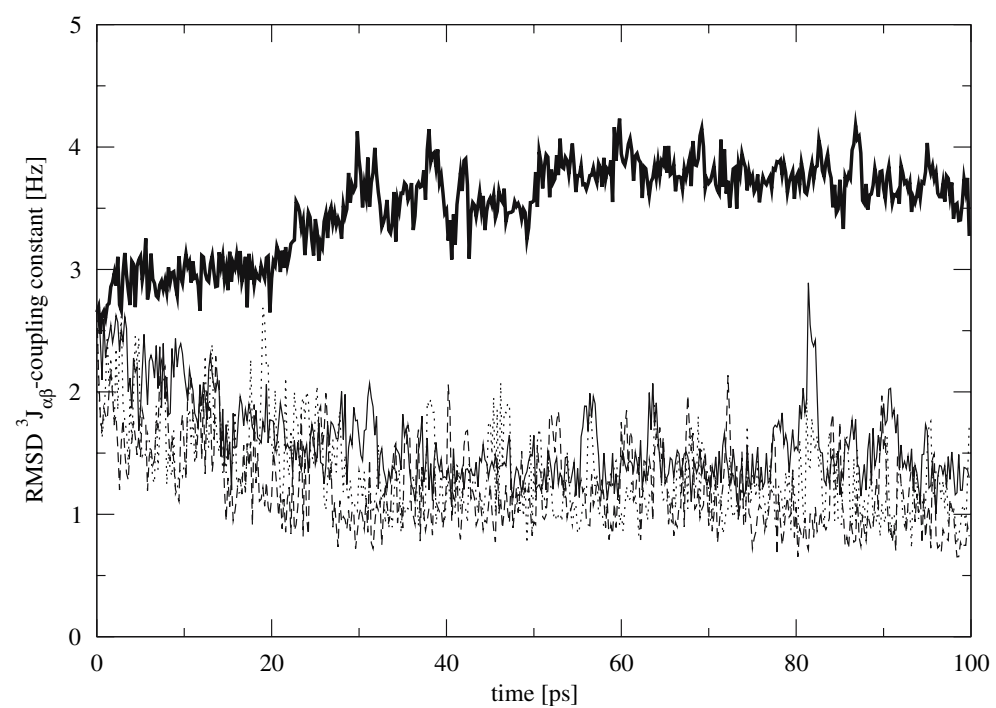

Fig. 2 Root-mean-square deviation (RMSD) of a set of 37 selected (see Table 1) ${ }^{3} J_{\alpha \beta}$-coupling constants from experimental values (Smith et al. 1991). The solid line is the root-mean-square deviation during an unrestrained simulation. All other lines are from simulations making use of local-elevation adaptive restraints. The thin line denotes use of a force constant $K^{\mathrm{Jres}}=0.005 \mathrm{~Hz}^{-4}$ and an allowed

only backbone atoms) decreases from $0.15 \mathrm{~nm}$ (unrestrained) to $0.12 \mathrm{~nm}$ in the restrained simulation (see Figs. 4 and 5). The atom-positional fluctuations are comparable. The secondary structure assignment shows no major loss in the overall structure of lysozyme, even though vacuum boundary conditions were used.

For the restrained simulation $\left(K^{\mathrm{Jres}}=0.005 \mathrm{~Hz}^{-4}, \Delta J^{0}=\right.$ $1.0 \mathrm{~Hz}$ and $N_{\mathrm{le}}=36$ ), a total of 1,630 inter-proton distances deviation of $\Delta J^{0}=1.0 \mathrm{~Hz}$, the dashed line use of a force constant $K^{\mathrm{Jres}}=0.1 \mathrm{~Hz}^{-4}$ and an allowed deviaton of $\Delta J^{0}=0.5 \mathrm{~Hz}$, the dotted line use of a force constant $K^{\mathrm{Jres}}=0.05 \mathrm{~Hz}^{-4}$ and acceptable deviation of $\Delta J^{0}=0.75 \mathrm{~Hz}$. All are using $N_{\mathrm{le}}=36$ intervals to discretise $\phi$, corresponding to a $\Delta \phi^{0}=10^{\circ}$

corresponding to NOE intensities (Soares et al. 2004; Schwalbe et al. 2001) have been analysed. We note that this set was the result of a slight revision (Soares et al. 2004) of a set of 1,632 NOE intensities (Schwalbe et al. 2001). These distance upper bounds include pseudo-atom corrections (Wüthrich et al. 1983) and the distances were determined from the simulations using $r^{-3}$ averaging (Tropp 1980). Their distribution is shown in Fig. 6 as 
Fig. 3 The local-elevation potential energy functions for four selected ${ }^{3} J_{\alpha \beta}$-coupling constants at the end of 100 ps of an adaptively restrained simulation $\left(K^{\mathrm{Jres}}=0.005 \mathrm{~Hz}^{-4}\right.$, $\Delta J^{0}=1.0 \mathrm{~Hz}$ and $\left.N_{\mathrm{le}}=36\right)$ are shown (Thr(51), $\operatorname{Thr}(69)$, $\operatorname{Thr}(89)$ and $\operatorname{Val}(109)$ corresponding to panel $\mathrm{A}, \mathrm{B}, \mathrm{C}$ and $\mathrm{D}$, upper half). The lower half depicts the time series (0.2 ps intervals) of the corresponding dihedral angle, black points indicate values obtained from the ${ }^{3} J$-value restraining simulation, red points those from an unrestrained simulation. The experimental and average ${ }^{3} J$-coupling constants are listed in Table 2

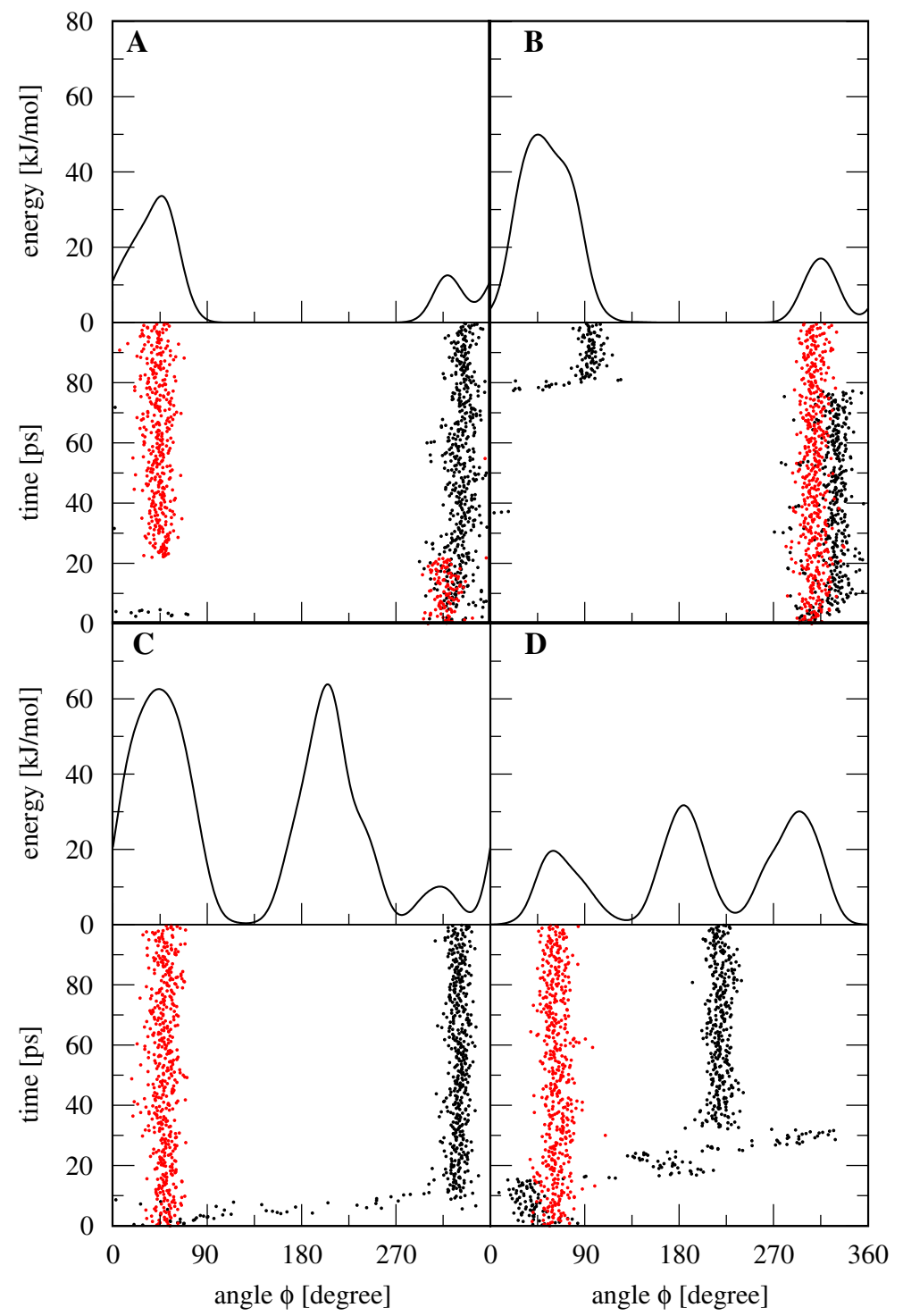

Table 2 Experimental (exp) ${ }^{3} J_{\alpha \beta}$-coupling constants (in $\mathrm{Hz}$; error about $1 \mathrm{~Hz}$ ) (Smith et al. 1991) and values obtained from $100 \mathrm{ps}$ of unrestrained (unrestr) and from $100 \mathrm{ps}$ of restrained (restr) simulation using adaptive (local-elevation) ${ }^{3} J$-value restraints with a set of 37 selected (see Table 1) ${ }^{3} J_{\alpha \beta}$-coupling constants for four residues that show large deviation between the values obtained from the free simulation and the experimental ones

\begin{tabular}{lccccc}
\hline Residue & & & \multicolumn{3}{l}{${ }^{3}$-coupling constant } \\
\cline { 5 - 6 } Name & Number & & Exp & Unrestr & Restr \\
\hline Thr & 51 & & 9.3 & 4.3 & 9.7 \\
Thr & 69 & & 9.3 & 12.5 & 9.8 \\
Thr & 89 & & 9.5 & 2.5 & 9.9 \\
Val & 109 & & 8.0 & 3.2 & 8.2 \\
\hline
\end{tabular}

distance bound violations, i.e. distances averaged over the simulation minus the corresponding NMR derived upper distance bound. This difference can also adopt negative values, which means that in the MD simulations the interproton distance is on average shorter than the upper bound derived from the NMR experiment. The black bars show the distribution of the simulation using ${ }^{3} J_{\alpha \beta}$-value restraining, shaded bars show the unrestrained distribution. Different from standard restraining simulations (using instantaneous or time-averaged ${ }^{3} J$-value restraints) less NOE violations are observed when using the adaptive (local-elevation) restraining method for ${ }^{3} J_{\alpha \beta}$-values presented here.

The dependence of the results on the initial structure of the simulation can be tested by repetitively using adaptive ${ }^{3} J$-coupling constant restraining followed by an unrestrained simulation period. Figure 7 shows that no improvement of the root-mean-square deviation of the ${ }^{3} J$-values during the unrestrained parts of the simulation is obtained. The simulations were carried out using a $20 \mathrm{ps}$ 
Fig. 4 The time series of secondary structure elements during an unrestrained simulation of lysozyme is shown

Fig. 5 The time series of secondary structure elements during a (local-elevation) ${ }^{3} J$-value restraining simulation using a set of $37{ }^{3} J$-coupling constant restraints with adaptive force constants of lysozyme using a force constant $K^{\mathrm{Jres}}=0.005 \mathrm{~Hz}^{-4}, \Delta J^{0}=1.0 \mathrm{~Hz}$ as acceptable deviation and $N_{\text {le }}=36$ is shown
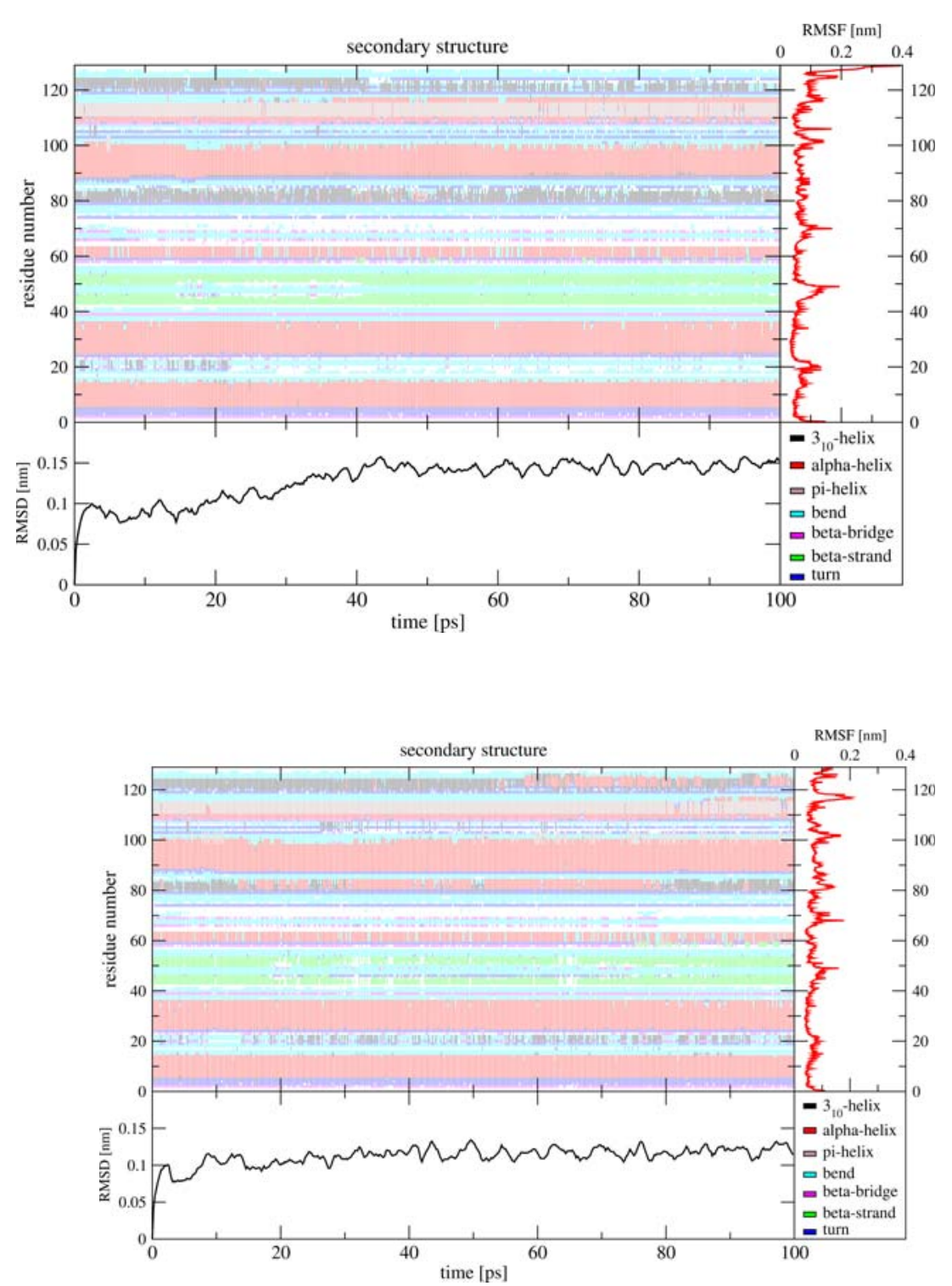

and a $40 \mathrm{ps}$ interval (with restraints switched on first, then switched off, etc.). The RMSD of ${ }^{3} J$-values immediately increases when the restraints are switched off. This may have two causes. First, even a 40 ps restraining period may not be enough to relax the structure (in vacuo), or, second, the force field does not properly favour the experimental conformation of the 37 side-chain angles and needs the adaptive restraining penalty function to correct for this error.

\section{Discussion}

A new application of the local-elevation simulation technique (Huber et al. 1994) to achieve ${ }^{3} J$-value restraining was presented. Using this method, it is possible to succesfully restrain ${ }^{3} J$-coupling constants without destabilising the overall molecular structure. In the example of lysozyme, even an improvement of reproducing experimental NOE distance bounds was observed. It can be applied to dihedral angles other than $\chi_{1}$, for which experimental ${ }^{3} J$-values are available (Perez et al. 2001).

The method achieves selectively enhanced sampling by disfavouring conformations of dihedral angles with ${ }^{3} J$-coupling constants deviating from experiment. Also, through the slow build up of the adaptive (local-elevation) potential energy penalty functions, a minimum of interference of the restraints compared to an unrestrained simulation is guaranteed. Furthermore, the method is not very sensitive with respect to the force constant and number of dihedral-angle intervals chosen, making it suitable to include ${ }^{3} J$-value restraining in standard biomolecular NMR structure refinement. 
Fig. 6 Violations of 1,630 NOE distance upper bounds from a 100 ps MD simulation of lysozyme are shown. Black bars correspond to a simulation using (local-elevation) ${ }^{3} J$-value restraining with $37{ }^{3} J_{\alpha \beta^{-}}$ coupling constant restraints $\left(K^{\mathrm{Jres}}=0.01 \mathrm{~Hz}^{-4}\right.$ and $\Delta J^{0}=1.0$ unrestrained simulation $\mathrm{Hz}$ ), white bars correspond to an

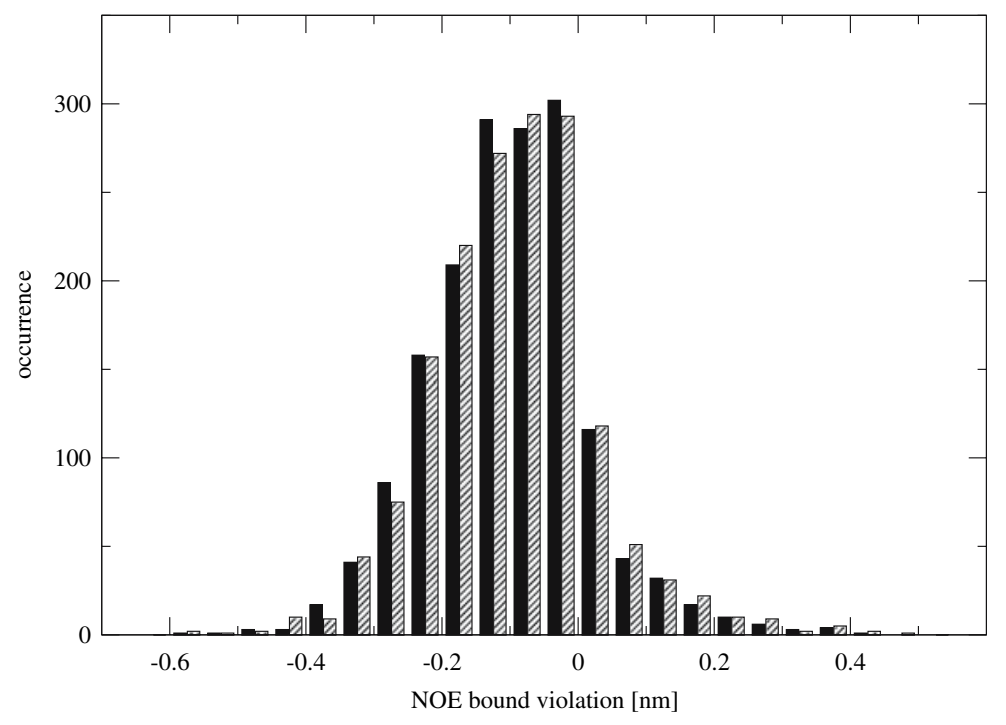

Fig. 7 The time series of the average root-mean-square deviation from the experimental ${ }^{3} J_{\alpha \beta}$-values for a set of 370

${ }^{3} J_{\alpha \beta}$-coupling constants is shown. In the simulation corresponding to the solid line, adaptive ${ }^{3} J$-coupling constant restraints $\left(K^{\mathrm{Jres}}=0.005 \mathrm{~Hz}^{-4}\right.$, $\Delta J^{0}=1.0 \mathrm{~Hz}$ and $N_{\text {le }}=36$ ) were switched off for the first $20 \mathrm{ps,}$ then on for $20 \mathrm{ps,} \mathrm{off} \mathrm{again} \mathrm{for}$ the next $20 \mathrm{ps}$ and so on. In the simulation denoted by the dotted black line, $40 \mathrm{ps}$ intervals were used

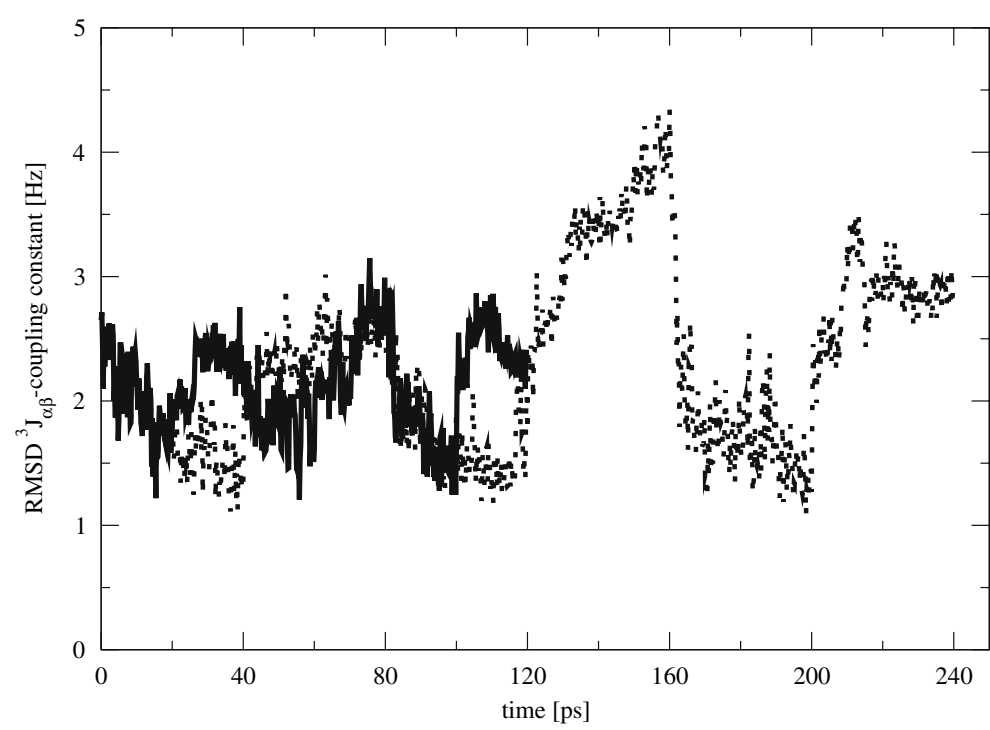

Jardetzky O (1980) Nature of molecular-conformations inferred from high-resolution NMR. Biochim Biophys Acta 621: 227-232

Kaptein R, Zuiderweg ERP, Scheek RM, Boelens R, van Gunsteren WF (1985) A protein-structure from nuclear magnetic-resonance data - Lac repressor headpiece. J Mol Biol 182:179-182

Karplus M (1959) Contact electron-spin coupling of nuclear magnetic moments. J Chem Phys 30:11-15

Markley JL, Bax A, Arata Y, Hilbers CW, Kaptein R, Sykes BD, Wright PE, Wüthrich K (1998) Recommendations for the presentation of NMR structures of proteins and nucleic acids IUPAC-IUBMB-IUPAB Inter-Union Task Group on the Standardization of Data Bases of Protein and Nucleic Acid Structures Determined by NMR Spectroscopy. J Biomol NMR 12:1-23

Nanzer AP, Poulsen FM, van Gunsteren WF, Torda AE (1994) A reassessment of the structure of Chymotrypsin inhibitor-2 (CI-2) using the time-averaged NMR restraints. Biochemistry 33: 14503-14511

Nanzer AP, van Gunsteren WF, Torda AE (1995) Parametrization of time-averaged distance restraints in MD simulations. J Biomol NMR 6:313-320 
Nanzer AP, Torda AE, Bisang C, Weber C, Robinson JA, van Gunsteren WF (1997) Dynamical studies of peptide motifs in the Plasmodium falciparum circumsporozoite surface protein by restrained and unrestrained MD simulations. J Mol Biol 267:1012-1025

Oostenbrink C, Villa A, Mark AE, van Gunsteren WF (2004) A biomolecular force field based on the free enthalpy of hydration and solvation: The GROMOS force-field parameter sets 53A5 and 53A6. J Comput Chem 25:1656-1676

Oostenbrink C, Soares TA, van der Vegt NFA, van Gunsteren WF (2005) Validation of the 53A6 GROMOS force field. Eur Biophys J 34:273-284

Pardi A, Billeter M, Wüthrich K (1984) Calibration of the angular dependence of the amide proton- $C_{\alpha}$ proton coupling-constants, ${ }^{3} J_{H N \alpha}$, in a globular protein. J Mol Biol 180:741-751

Perez C, Göhr F, Rüterjans H, Schmidt JM (2001) Self-consistent Karplus parametrization of ${ }^{3} J$ couplings depending on the polypeptide side-chain torsion $\chi_{1}$. J Am Chem Soc 123: 7081-7093

Ryckaert JP, Ciccotti G, Berendsen HJC (1977) Numerical-integration of Cartesian equation of motion of a system with constraints - molecular-dynamics of n-alkanes. J Comput Phys 23:327-341

Schmidt JM, Blümel M, Löhr F, Rüterjans H (1999) Self-consistent ${ }^{3} J$ coupling analysis for the joint calibration of Karplus coefficients and evaluation of torsion angles. J Biomol NMR 14:1-12

Schuler LD, Daura X, van Gunsteren WF (2001) An improved GROMOS96 force field for aliphatic hydrocarbons in the condensed phase. J Comput Chem 22:1205-1218

Schwalbe H, Grimshaw SB, Spencer A, Buck M, Boyd J, Dobson CM, Redfield C, Smith LJ (2001) A refined solution structure of hen lysozyme determined using residual dipolar coupling data. Protein Sci 10:677-688

Scott WRP, Hünenberger PH, Tironi IG, Mark AE, Billeter SR, Fennen J, Torda AE, Huber T, Krüger P, van Gunsteren WF (1999) The GROMOS biomolecular simulation program package. J Phys Chem A 103:3596-3607
Scott WRP, Mark AE, van Gunsteren WF (1998) On using timeaveraging restraints in molecular dynamics simulation. J Biomol NMR 12:501-508

Smith LJ, Sutcliffe MJ, Redfield C, Dobson CM (1991) Analysis of the $\phi$ and $\chi_{1}$ torsion angles for hen lysozyme in solution from ${ }^{1} H$-NMR spin spin coupling-constants. Biochem 30:986-996

Soares TA, Daura X, Oostenbrink C, Smith LJ, van Gunsteren WF (2004) Validation of the GROMOS force-field parameter set 45A3 against nuclear magnetic resonance data of hen egg lysozyme. J Biomol NMR 30:407-422

Torda AE, Scheek RM, van Gunsteren WF (1989) Time-dependent distance restraints in molecular-dynamics simulations. Chem Phys Lett 157:289-294

Torda AE, Brunne RM, Huber T, Kessler H, van Gunsteren WF (1993) Structure refinement using time-averaged $J$-coupling constant restraints. J Biomol NMR 3:55-66

Tropp J (1980) Dipolar relaxation and nuclear Overhauser effects in nonrigid molecules - the effect of fluctuating inter-nuclear distances. J Chem Phys 76:6035-6043

van Gunsteren WF, Billeter SR, Eising AA, Hünenberger PH, Krüger P, Mark AE, Scott WRP, Tironi IG (1996) Biomolecular simulation: The GROMOS96 manual and user guide. Verlag der Fachvereine, Zürich

van Gunsteren WF, Bonvin AMJJ, Daura X, Smith LJ (1999) Structure Computation and Dynamics in Protein NMR. In: Krishna RM, Berliner LJ (eds) Chap. aspects of modeling biomolecular structure on the basis of spectroscopic or diffraction data, vol 17. Plenum Publishers, New York, pp 3-35

Wang AC, Bax A (1996) Determination of the backbone dihedral angles $\phi$ in human ubiquitin from reparametrized empirical Karplus equations. J Am Chem Soc 118:2483-2494

Wüthrich K, Billeter M, Braun W (1983) Pseudo-structures for the 20 common amino-acids for use in the studies of protein conformations by measurements of intramolecular proton proton distance constraints with nuclear magnetic-resonance. J Mol Biol 169:949 